\title{
An attribute frequency model for the abstraction of prototypes
}

\author{
PAUL G. NEUMANN \\ University of Colorado, Boulder, Colorado 80302
}

\begin{abstract}
An attribute frequency model for the abstraction of prototypes is proposed as an alternative to the prototype-plus-transformation model. A specific model is tested in a Franks and Bransford visual pattern paradigm under conditions in which the two models generate contrasting predictions. The results support the attribute frequency model. Application of the model to reported data obtained in other paradigms is illustrated and discussed.
\end{abstract}

Studies of concept formation have traditionally used concepts defined by a listing of relevant attributes (Bourne, 1966). Recently, Franks and Bransford (1971) have proposed that concepts might more generally be defined in terms of a base pattern, or prototype, plus an allowable set of transformations. In their experiments, the base pattern consisted of a particular arrangement of four discrete, discriminable visual attributes. A set of formal transformation rules was defined, and distortions of the prototype were produced by varying the prototypical pattern in accordance with various combinations of these rules. An acquisition set was assembled with the restriction that each type of distortion defined by the transformation rules was represented and that the prototype remain the "central tendency" of the acquisition set. The central tendency of the prototype is ensured by determining that the total number of individual transformations required to produce representations of all transformations defined by the set of rules is least when the prototype is used as the base pattern.

Following exposure to the acquisition set, Ss were exposed to a recognition set and told to decide whether they had seen each pattern in the acquisition set and to rate their confidence on a 5-point scale. The results indicate that the prototype (generally not included in the acquisition set) received the highest positive (recognized) rating, followed by patterns of increasing transformational distance. Patterns that could not be produced by the formal transformation rules tended to receive negative (not recognized) ratings. These results were interpreted as supportive of a schematic memory structure for concepts consisting of a prototype and a set of transformation rules that produce instances of the concept.

*This research was supported by Grants GB $34077 \mathrm{X}$ to this Institute and GJ 453 to the Computer Laboratory for Instruction in Psychological Research, both from the National Science Foundation, Grant MH 14314 to Lyle E. Bourne, Jr. from the National Institute of Mental Health, and by an NDEA graduate fellowship awarded to the author, who wishes to express his gratitude for the judicious combination of support and criticism offered by Lyle E. Bourne, Ir., who decimated an earlier version of this manuscript. This is Publication 39 of the Institute for the Study of Intellectual Behavior. Requests for reprints should be sent to Paul G. Neumann, Institute for the Study of Intellectual Behavior, University of Colorado, Boulder, Colorado 80302.
The nature of the experimental paradigm used by Franks and Bransford would seem to make these results equally (or more) amenable to the predictions of an attribute frequency model. The general model proposed here associates each attribute of the stimulus with a set of frequency counts, one for each discriminable state of each dimension upon which the aitribute varies. In addition, when specific cues are present which define relationships among attributes, an additional frequency count is associated with each discriminable relationship between attributes consistent with these cues.

Frequency counts are compiled from the acquisition stimuli to be shown Ss. The number of appearances of each value of each dimension upon which the attribute varies is counted. For example, if the attribute triangle varies on the dimensions size and color, the number of times each size occurs and the number of times each color appears is counted. These counts will be referred to as dimension state frequencies.

In addition, when cues to the relevance of relationships between attributes are present, a count is made of the number of appearances of each of the cued relationships. In the above example, counts may be made for large-red, large-green, small-red, and small-green. These counts will be referred to as relational state frequencies.

In its present state of development, the model makes no formal assumptions as to the processes by which the frequency information is coded, stored, retrieved, or evaluated by S. It is only assumed that through some process or combination of processes the frequencies of the dimension states and cued relational states affect the recognition process in the following predictable manner. When a pattern is presented to $S$ for recognition, if that pattern incorporates one or more relational states that did not occur in the acquisition patterns, the recognition pattern will be judged as not having occurred in the acquisition set. Note that a nonoccurring attribute automatically generates nonoccurring relationships. In the absence of cued relationships, it is assumed that a nonoccurring attribute will also result in the pattern being judged as not having occurred in the acquisition set. Therefore, any attribute or cued relationship among attributes associated with a frequency count of zero predicts nonrecognition of a pattern.

The role of the dimension state frequencies is in determining the confidence of the rating. If the pattern 
in question is judged to have occurred in the acquisition set, high dimension state frequencies produce high confidence ratings and low dimension state frequencies produce low confidence ratings. If the pattern is judged not to have occurred in the acquisition set, the opposite result obtains. High dimension state frequencies produce low negative ratings, and low dimension state frequencies produce high negative ratings. Thus, greater dimension state frequencies tend to bias the rating in a positive direction. The order of the final ratings is predicted, from highest to lowest, by the order of the sums of the dimension state frequencies for each recognition pattern, beginning with those for which there is no nonoccurring attribute or relational state, exhausting this set, then continuing with those for which one or more attributes or relational states were nonoccurring in the acquisition set. The following experiments were designed to test the viability of such a model in comparison with the predictions of the Franks and Bransford prototype-plus-transformation model.

\section{EXPERIMENT I}

In Experiment III of Franks and Bransford (1971), the stimuli consisted of square cards bearing a unique visual attribute in each of the four quadrants. A horizontal line separated the upper and lower halves of the card. Two prototypes were constructed that were identical, with the exception of the shape of one attribute. An acquisition set was then constructed by distorting the prototypes in accordance with rules that permitted upper-lower exchange of horizontal pairs, left-right exchange of attributes within a pair, and deletion of one attribute. The experimental procedure was consonant with the previous general description of the paradigm and will be more fully elaborated in the Method sections that follow. The purpose of this experiment was to contrast the predictions of a simple frequency model based upon the frequencies with which individual attributes occupied each quadrant across the series of patterns in the acquisition set with predictions of the prototype-plus-transformation model. Comparisons were made between ratings of stimuli for which the sums of the frequencies were equal but for which there was a difference in the number of transformations required to produce the two patterns. The results favored the prototype-plus-transformation model over the simple frequency model.

In their Experiment IV, Franks and Bransford added six stimuli to the recognition set. These stimuli could not be produced by the transformation rules but, nevertheless, incorporated combinations of the same visual attributes from which the other stimuli were constructed. The simple frequency model predicted recognition of these patterns commensurate with the sums of the individual frequencies, whereas the prototype-plus-transformation model predicted nonrecognition of these stimuli. The results, once again, favored the prototype-plus-transformation model.

Experiment I of the present series was a replication of Franks and Bransford's (1971) Experiment IV, intended to ensure the comparability of the procedures used.

\section{Method}

\section{Subjects}

Ss were 18 undergraduate psychology students who volunteered for participation in partial fulfillment of optional course requirements.

\section{Materials}

The stimuli were reproductions of those illustrated in Franks and Bransford (1971, Fig. 2). They consisted of square white cards divided into upper and lower halves by a horizontal line. The attributes consisted of a heart, a triangle, a square, a cross, and a circle. Each stimulus had either three or four of these attributes distributed among the four quadrants in accordance with the transformation rules applied to one of the two prototypes. The attributes were roughly equal in area, were centered within the quadrants, and were colored black. The stimuli were photographed on a black background for projection. Large white cards with a horizontal line separating the top and bottom halves and reproductions of the five attributes were constructed for Ss' reproduction task.

\section{Procedure}

Ss were given the reproduction materials and were told that they would participate in a short-term memory task. They were instructed to view carefully each slide presented by E. After the pattern disappeared from the screen, they were to count from one to five with $\mathrm{E}$, then reproduce the pattern with the materials provided.

Patterns were projected for $5 \mathrm{sec}$, followed by a dark screen. After counting from one to five, E checked the reproductions and directed a reconfiguration when an error was made until the pattern was correct. The acquisition set was projected twice, first in a random order and then in the reverse of that order. A 5-min break followed the acquisition sequence, during which the reproduction materials were collected and the recognition rating sheets were distributed. Ss were told that they would see a sequence of patterns, some of which had appeared in the first series of patterns and some of which had not. They were instructed to decide whether each pattern had appeared in the first sequence and to mark how sure they were on a 10-point scale that was discontinuous at 0 . The possible ratings, thus, ran from +1 to +5 and -1 to -5 . The recognition set was projected in a random order at a 4-sec rate. To minimize serial errors when $S s$ marked the ratings, $E$ announced the order number of each slide as it was projected. At the end of the first recognition sequence, there was a 2-min break, during which $\mathrm{E}$ collected the first rating sheet. The recognition set was then projected in the reverse order, and Ss rated the patterns a second time to offset any effects of frequency counțs that might accumulate during exposure to the recognition set.

\section{Results}

Mean ratings were compiled by transformational distance. Table 1 indicates that the prototype received the highest recognition rating, followed by patterns of increasing transformational distance. A crossover in the predicted order occurred between patterns of Transformation Distances 3 and 4, but the "noncases" 
received a negative mean rating, indicating that they were not "recognized."

\section{Discussion}

Although not an exact replication, the essential results of Franks and Bransford (1971, Experiment IV) were obtained. In general, the means were ordered by transformational distance and the mean of the noncases was negative, indicating that the patterns that could not be produced by the rules were not evaluated as members of the acquisition set.

A specific application of the present attribute frequency model to this paradigm is as follows. The locus of each visual attribute varies on two planes, horizontal and vertical, and each plane incorporates two positions, left-right and upper-lower, respectively. There are, therefore, two dimensions on which the attributes vary, and each dimension incorporates two states. Each attribute is, therefore, associated with a dimension state frequency for left, one for right, one for upper, and one for lower. It is assumed that there are actually six attributes. A circle, a triangle, a cross, a heart, and a square comprise the first five. Deletion of any one of these attributes produces the sixth visual attribute, a blank quadrant.

The horizontal line separating the top and bottom halves of the card is an explicit cue to the horizontal pairing of attributes. Therefore, relational state frequencies are associated with the left-right relationships for each possible pairing. Table 2 lists the frequency counts derived from the acquisition set in this manner. Note that the greater frequencies occur in the dimension states and relational states represented by the prototypes.

To determine the predicted order of the mean recognition ratings, the relational state frequencies of a pattern are first consulted. If all relational state frequencies are nonzero, it is assumed that the pattern will be "recognized." If one or more are zero, it is assumed that the pattern will "not be recognized." The magnitude of the positive or negative rating is then ascertained by summing the dimension state frequencies for each pattern. For example, one of the prototypes consists of a circle in the upper left, a triangle in the upper right, a heart in the lower left, and a square in the

Table 1

Mean Recognition Ratings for Experiment I

\begin{tabular}{ccc}
\hline $\begin{array}{c}\text { Transfor- } \\
\text { mation } \\
\text { Distance }\end{array}$ & $\begin{array}{c}\text { Mean } \\
\text { Rating }\end{array}$ & SD \\
\hline 0 & 2.79 & .78 \\
1 & 2.18 & .98 \\
2 & .49 & 1.44 \\
3 & .90 & 1.04 \\
Noncase & -.26 & 1.82 \\
\hline
\end{tabular}

Table 2

Dimension State and Nonzero Relation State Frequencies for the Acquisition Set

\begin{tabular}{|c|c|c|c|c|}
\hline \multirow[b]{2}{*}{ Attribute } & \multicolumn{4}{|c|}{ Dimension State Frequencies } \\
\hline & Upper & Lower & Left & Right \\
\hline Circle & $8^{*}$ & 4 & $8^{*}$ & 4 \\
\hline Triangle & $4 *$ & 2 & 2 & $4^{*}$ \\
\hline Cross & $4^{*}$ & 2 & 2 & $4^{*}$ \\
\hline Heart & 3 & $6^{*}$ & $6^{*}$ & 3 \\
\hline Square & 3 & $6^{*}$ & 3 & $6^{*}$ \\
\hline Blank & 2 & 4 & 3 & 3 \\
\hline \multicolumn{5}{|c|}{ Relational State Frequencies } \\
\hline Relationship & Frequency & Rela & & Frequency \\
\hline Circle-Triangle & $4^{*}$ & Trian & & 2 \\
\hline Circle-Cross & $4^{*}$ & Cross & & 2 \\
\hline Heart-Square & $4^{*}$ & Squar & & 2 \\
\hline Heart-Blank & 2 & Blank & & 1 \\
\hline Square-Blank & 1 & Blank & & 2 \\
\hline
\end{tabular}

*Denotes state represented by prototype

Table 3

Summed Dimension State Frequencies by Transformational Distance

\begin{tabular}{cccl}
\hline Distance & Pattern & Frequency & \multicolumn{1}{c}{ Description } \\
\hline 0 & 1,9 & 48 & $\begin{array}{l}\text { Prototype } \\
\text { Upper-Lower Exchange } \\
1\end{array}$ \\
2,10 & 36 & $\begin{array}{l}\text { Right-Left Exchange } \\
\text { Upper Pair } \\
\text { Right-Left Exchange } \\
\text { Lower Pair } \\
\text { Deletion of One Attribute } \\
\text { (Square or Heart) } \\
\text { Upper-Lower Exchange } \\
\text { and Deletion } \\
\text { Right-Left Exchange } \\
\text { Both Pairs } \\
\text { Right-Left Exchange Both } \\
\text { Pairs and Deletion }\end{array}$ \\
\hline
\end{tabular}

lower right. The dimension state frequency for circle-upper is 8 , for circle-left is 8 , for triangle-upper is 4 , for triangle-right is 4 , for heart-lower is 6 , for heart-left is 6 , for square-lower is 6 , and for square-right is 6 . The sum of these frequencies is 48 . Table 3 lists the sum of these frequencies so obtained and a description of the transformations that produce these patterns. In addition to these patterns, a set of six "noncases" is defined by one or more zero relational frequencies. This set is isomorphic to the set of "noncases" defined within the framework of the prototype-plus-transformation model and has a mean dimension state frequency sum of 39.2 .

The results of Experiment I may be compared to the predictions of this model. In general, the model predicts a mean rating with decreases with increasing transformational distance as defined by the prototype-plus-transformation model (cf. Table 3). A discrepancy exists in that the mean dimension state 
Table 4

A Comparison of Predictions of the Prototype-PlusTransformation Model, Simple Frequency Model, and Attribute Frequency Model for Specific Pattern Pairs

\begin{tabular}{cccc}
$\begin{array}{c}\text { Patterns } \\
\text { Compared }\end{array}$ & $\begin{array}{c}\text { Prototype } \\
\text { Plus Trans- } \\
\text { formation }\end{array}$ & $\begin{array}{c}\text { Simple } \\
\text { Frequency } \\
\text { Model }\end{array}$ & $\begin{array}{c}\text { Present } \\
\text { Frequency } \\
\text { Model }\end{array}$ \\
\hline 1 vs 2 & $1_{0}>2_{1}^{*}$ & $1_{12}=2_{12}$ & $1_{48}>2_{36}$ \\
1 vs 3 & $1_{0}>3_{1}$ & $1_{12}=3_{12}$ & $1_{48}>3_{42}$ \\
1 vs 4 & $1_{0}>4_{1}$ & $1_{12}=4_{12}$ & $1_{48}>4_{42}$ \\
1 vs 7 & $1_{0}>7_{2}$ & $1_{12}=7_{12}$ & $1_{48}>7_{36}$ \\
3 vs 7 & $3_{1}>7_{2}$ & $3_{12}=7_{12}$ & $3_{42}>7_{36}$ \\
4 vs 7 & $4_{1}>7_{2}$ & $4_{12}=7_{12}$ & $4_{42}>7_{36}$ \\
5 vs 6 & $5_{1}>6_{2}$ & $5_{9}=6_{9}$ & $5_{43}>6_{32}$ \\
5 vs 8 & $5_{1}>8_{3}$ & $5_{9}=8_{9}$ & $5_{43}>8_{34}$ \\
9 vs 10 & $9_{0}>10_{1}$ & $9_{12}=10_{12}$ & $9_{48}>10_{36}$ \\
9 vs 11 & $9_{0}>11_{1}$ & $9_{12}=11_{12}$ & $9_{48}>11_{42}$ \\
9 vs 12 & $9_{0}>12_{1}$ & $9_{12}=12_{12}$ & $9_{48}>12_{42}$ \\
9 vs 15 & $9_{0}>15_{2}$ & $9_{12}=15_{12}$ & $9_{48}>15_{36}$ \\
11 vs 15 & $11_{1}>15_{2}$ & $11_{12}=15_{12}$ & $11_{42}>15_{36}$ \\
12 vs 15 & $12_{1}>15_{2}$ & $12_{12}=15_{12}$ & $12_{42}>15_{36}$ \\
13 vs 14 & $13_{1}>14_{2}$ & $13_{9}=14_{9}$ & $13_{43}>14_{32}$ \\
13 vs 16 & $13_{1}>16_{3}$ & $13_{9}=16_{9}$ & $13_{43}>16_{34}$ \\
\hline
\end{tabular}

*Subscript indicates transformation distance or summed frequency.

frequencies are equal for a transformational distance of 2 and 3 . Table 1 indicates that the obtained mean for a transformational distance of 3 is slightly higher than that obtained for a transformational distance of 2 , which is more compatible with the prediction of the attribute frequency model, which predicts equality, than the prototype-plus-transformation model, which predicts the opposite order. In addition, the mean rating for the noncases was low negative, as predicted by the distribution of frequencies.

The predictions of the present model may also be applied to the specific pairs of patterns that Franks and Bransford used to contrast the predictions of the prototype-plus-transformation model with those of the simple frequency model. Table 4 illustrates that, although the simple frequency model predicts equality for the pairings, the present model predicts the same inequality predicted by the prototype-plustransformation model.

Thus far, the evidence presented merely indicates that the present model is capable of generating essentially the same predictions as the prototype-plus-transformation model. To contrast the two models directly, stimuli were constructed that were identical to the stimuli used in Experiment I, with the exception that the horizontal line dividing the upper and lower halves of the cards was removed. This has differential effects within the framework of the two models. Within the context of the attribute frequency model, its effect is to remove the pairing cues. Therefore, the relational frequencies with which the attributes appear in each left-right relationship are eliminated. This in turn destroys the distinction between "cases" and "noncases." All patterns should receive positive ("recognized") ratings based solely on the sums of the dimension state frequencies.
Within the context of the prototype-plustransformation model, the possible effects are more diverse. The removal of the horizontal line creates three schemata that might be derived from the variation among stimuli. The first is the one by which the patterns were actually produced, based on the horizontal pairing of attributes. The second is based on vertical pairing of the attributes. This schema would create changes in the predicted order of the mean ratings, since a pattern based on upper-lower exchange of horizontally defined pairs is now equivalent to two attribute exchanges within vertically defined pairs, as illustrated in Fig. 1. In addition, two "noncases" become "cases," and four "cases" become "noncases." The third schema is based on the absence of any pair relationships. The transformational rules would permit the exchange of any two adjacent attributes plus the deletion of an attribute. Table 5 lists the transformational distances obtained within the framework of all three possible schemata.

\section{EXPERIMENT II}

Experiment II was designed to contrast the predictions of the three schemata generated by the prototype-plus-transformation model with the predictions of the attribute frequency model under conditions in which there are no visual cues to the pairing of attributes. The prototype-plus-transformation model predicts ordering of the mean recognition ratings consistent with one of the three generated schemata, whereas the attribute frequency model predicts ordering

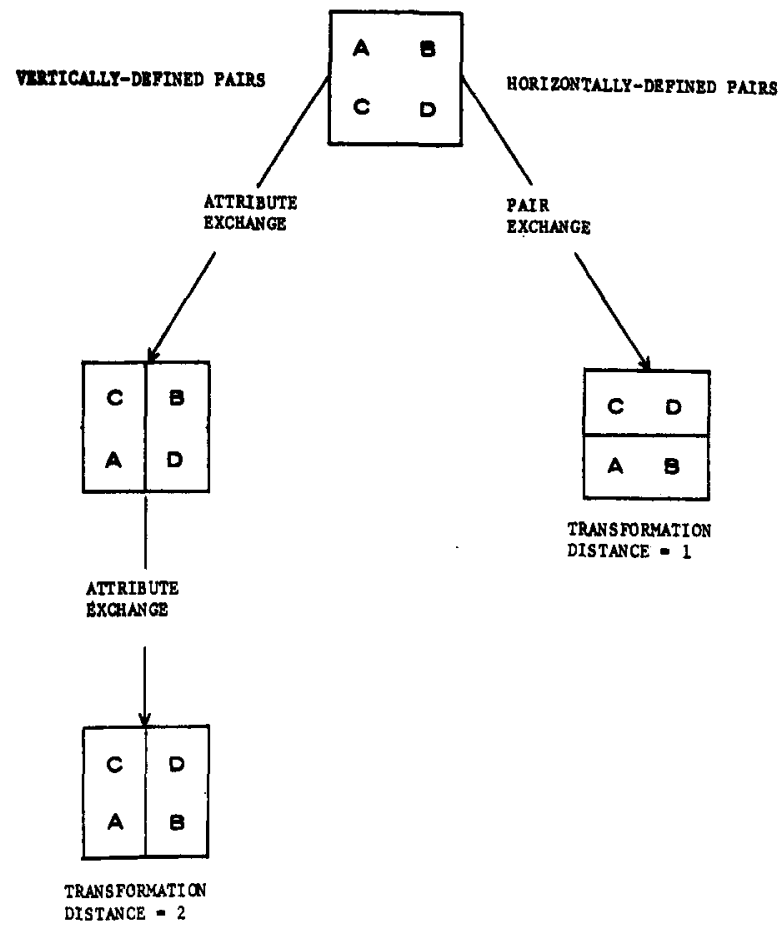

Fig. 1. Production of identical patterns of different transformational distance by two schemata. 
based solely on the summed dimension state frequencies, with no negative mean ratings (i.e., no "noncases").

\section{Method}

The procedure and materials were identical to that used for Experiment I, with the exception that the horizontal line on the projected patterns and on the reproduction materials was removed. Ss were 27 undergraduate psychology students obtained in the manner described for Experiment $I$.

\section{Results}

Mean ratings were compiled by transformational distance for all three schemata and by summed dimension state frequencies. Only one "noncase" received a negative mean rating $(\overline{\mathrm{X}}=-1.41, \mathrm{SD}=3.06)$. This pattern is listed as Noncase 4 in Table 5 and, thus, is not the single noncase within the framework of the no-pairs schema. Table 6 lists the means by transformational distance for all three schemata and by the summed dimension state frequencies. Concordance of order tests (Kendall's tau) were performed with identical results for the schema based upon vertical pairs, the schema based upon horizontal pairs, and the schema based upon no pairs $(z=1.58, p<.06)$. Thus, the results of the test failed to reject the hypothesis of no concordance by a narrow margin. For the order by dimension state frequencies, the results were much less ambiguous $(\mathrm{z}=2.38, \mathrm{p}<.01)$, indicating a significant concordance of the sums of the dimension state frequencies and the order of the means.

\section{Discussion}

The results of Experiment II demonstrate that the attribute frequency model is superior to the three schemata obtained from the prototype-plustransformation model in predicting the results of a visual pattern recognition experiment in the Franks and Bransford paradigm, when visual cues as to the pairings of attributes are not present. All three schemata predicted negative mean ratings for one or more noncases, which are defined as patterns that cannot be
Table 5

Transformation Distances Derived from the Three Schemata

\begin{tabular}{|c|c|c|c|}
\hline & $\begin{array}{l}\text { Horizontal } \\
\text { Pairing }\end{array}$ & $\begin{array}{l}\text { Vertical } \\
\text { Pairing }\end{array}$ & $\begin{array}{c}\text { No } \\
\text { Pairing }\end{array}$ \\
\hline \multicolumn{4}{|l|}{ Pattern } \\
\hline 1,9 & 0 & 0 & 0 \\
\hline 2,10 & 1 & 2 & 2 \\
\hline 3,11 & 1 & $\mathrm{NC}^{*}$ & 1 \\
\hline 4,12 & 1 & $\mathrm{NC}^{*}$ & 1 \\
\hline 5,13 & 1 & 1 & 1 \\
\hline 6,14 & 2 & 3 & 3 \\
\hline 7,15 & 2 & 1 & 2 \\
\hline 8,16 & 3 & 2 & 3 \\
\hline \multicolumn{4}{|l|}{ Noncase } \\
\hline 1 & $\mathrm{NC}^{*}$ & 1 & 1 \\
\hline 2 & $\mathrm{NC}^{*}$ & 1 & 1 \\
\hline 3 & $\mathrm{NC}^{*}$ & $\mathrm{NC}^{*}$ & 3 \\
\hline 4 & $\mathrm{NC}^{*}$ & $\mathrm{NC}^{*}$ & 3 \\
\hline 5 & $\mathrm{NC}^{*}$ & $\mathrm{NC}^{*}$ & 3 \\
\hline 6 & $\mathrm{NC}^{*}$ & $\mathrm{NC}^{*}$ & $\mathrm{NC}^{*}$ \\
\hline
\end{tabular}

*Noncase

produced within the framework of the schema. The mean ratings of these patterns, with one exception, were decidedly positive. The pattern that received the negative rating was not the pattern predicted to be a noncase by the schema which predicts only one such pattern (the no-pairs schema).

The attribute frequency model predicts that these patterns should receive positive ratings, since the exclusion of relational state frequencies, which are the basis upon which a pattern may be ascertained not to have been included in the acquisition set, prevents negative ratings. Ratings are, therefore, based entirely upon the sums of dimension state frequencies. Concordance of order tests support the latter prediction over the three former predictions.

\section{EXPERIMENT III}

Experiment III was designed to determine whether the effects of the pairing cues are greater when included on the stimuli themselves or on the reproduction materials given to Ss.

Table 6

Mean Ratings for Experiment II

\begin{tabular}{|c|c|c|c|c|c|c|c|c|c|c|c|}
\hline TD* & & & $\mathrm{TD}^{*}$ & & & $\mathrm{TD}^{*}$ & & & & & \\
\hline $\begin{array}{l}\text { Horl- } \\
\text { zontal } \\
\text { Pairing }\end{array}$ & Mean & $\mathrm{SD}$ & $\begin{array}{l}\text { Vertical } \\
\text { Pairing }\end{array}$ & Mean & $\mathrm{SD}$ & $\begin{array}{c}\text { No } \\
\text { Pairing }\end{array}$ & Mean & SD & $\begin{array}{c}\text { Fre- } \\
\text { quency }\end{array}$ & Mean & SD \\
\hline 0 & 2.36 & .73 & 0 & 2.36 & .73 & 0 & 2.36 & .73 & 48 & 2.36 & .73 \\
\hline 1 & 1.89 & .77 & 1 & 1.36 & .99 & 1 & 1.81 & .73 & 43 & 1.73 & .63 \\
\hline 2 & .56 & .72 & 2 & .95 & 1.87 & 2 & 1.42 & 1.22 & 42 & 1.66 & .93 \\
\hline 3 & -.71 & 1.16 & 3 & .90 & .88 & 3 & .05 & 1.25 & 41 & 1.54 & 1.46 \\
\hline Noncase & .84 & 1.47 & Noncase & .99 & 1.30 & Noncase & 1.54 & 1.24 & 36 & .73 & 1.49 \\
\hline & & & & & & & & & 34 & .14 & 1.33 \\
\hline & & & & & & & & & 32 & .90 & .88 \\
\hline
\end{tabular}


Table 7

Mean Ratings for Experiment III

\begin{tabular}{lccccc}
\hline $\begin{array}{l}\text { Transfor- } \\
\text { mational } \\
\text { Distance* }\end{array}$ & Mean & SD & $\begin{array}{c}\text { Fre- } \\
\text { quency }\end{array}$ & Mean & SD \\
\hline 0 & 2.58 & .40 & 48 & 2.58 & .40 \\
1 & 2.20 & .36 & 43 & 2.34 & .49 \\
2 & 1.23 & 1.07 & 42 & 2.16 & .73 \\
3 & .17 & .23 & 41 & 2.21 & .33 \\
Noncase & 1.50 & 1.06 & 36 & 1.73 & 1.02 \\
& & & 34 & .47 & .67 \\
& & & 32 & .31 & .57 \\
\hline
\end{tabular}

*Horizontal pairings

\section{Method}

The procedure and materials were identical to those in Experiment I, with the exception that the horizontal line dividing the upper and lower halves of the pattern was retained on the slides but deleted on the reproduction materials. Ss were 24 undergraduate psychology students obtained in the manner described for Experiment I.

\section{Results}

All noncases received positive ratings. Table 7 indicates a single crossover in the predicted order by summed dimension state frequencies. This crossover occurred between patterns that differ in summed frequencies by only one occurrence of an attribute ( 42 vs 41 ), and the difference in the mean rating was only .05. A concordance of order test on the ordering by dimension state frequencies rejects the no-concordance hypothesis $(\mathrm{z}=2.72, \mathrm{p}<.01)$.

\section{Discussion}

The primary effect of the visual cues to pairing relationships apparently lies in being present on the reproduction materials, since the results of Experiment III are nearly identical to those of Experiment II, with the exception of the locus of the crossover in predicted order and the fact that the mean ratings are generally higher for Experiment III than for Experiment II. The restoration of the horizontal line on the slides had little effect. This is not surprising in view of the relative duration of exposure to the slides $(5 \mathrm{sec})$ and to the reproduced pattem (approximately $40 \mathrm{sec}$ ), during which $E$ checks the validity of the reproductions. Experiment III again demonstrates that the attribute frequency model successfully predicts the order of mean ratings of the patterns.

\section{GENERAL DISCUSSION}

The experiments reported here have contrasted the predictions of the prototype-plus-transformation model with the predictions of a specific model derived from a general frequency theory of attribute abstraction and concept learning. The prototype-plus-transformation model assumes that concept-relevant information consists of the variation between patterns, from which the identity of the rules by which the prototype is distorted may be abstracted. The identity of the prototype itself may then be established by applying the inverse of these rules to the distortions. The attribute frequency model, in contrast, assumes that the prototype receives the highest recognition ratings because the dimension state and relational state frequencies are highest for those states that are represented by the prototype. In the Franks and Bransford paradigm, this is essentially an artifact of the restriction that the prototype remain the central tendency of the patterns represented in the acquisition set.

There are several serious defects in the prototype-plus-transformation approach. First, the theory imposes a great memory load upon S, who must recall the patterns, determine the variation, and derive the rules, all, presumably, in his head. This assumption is not supported by some of the results reported by Franks and Bransford (1971), who concluded that there was little evidence for memory of specific patterns. Assuming, however, that $\mathrm{S}$ is capable of dealing with both the memory load and the information processing load, it is not clear how $S$ determines which rules have been applied to a specific pattern in order to perform the inverse transformations and determine the identity of the prototype. Equally unclear is the restriction that the prototype remain the central tendency of the patterns included in the acquisition set. If $S$ is capable of abstracting a rule from one pattern of a Distance 3 transformation, he should be at least equally capable of making the abstraction from two of three instances of distortions of this magnitude.

In contrast to the prototype-plus-transformation model, the attribute frequency model does not formally assume memory for complete patterns or for variations among patterns. It further requires no assumptions about processing of information, abstraction of rules, or identity of the prototype. It is based on the assumption that the more often a particular state of an attribute or state of a relationship between attributes occurs, the more likely $\mathrm{S}$ is to remember its having occurred. The frequency with which discriminable states of attributes and relationships among attributes occur is considered to be a predictor of the confidence with which $S$ recognizes a pattern that includes certain of these states. The success of this psychologically simpler theory in a specific application was demonstrated in direct contrast to the predictions of the prototype-plus-transformation model.

In a recent publication, Reitman and Bower (1973) propose a theory accounting for results obtained with patterns composed of linearly distributed elements. This theory assumes that the information stored is a series of tags on each element and combination of elements, one tag for each occurrence in the acquisition set. During 
recognition, the first pass considers the number of tags on each individual element. Each time the number of tags on an element exceeds a threshold value, a tally counter is incremented. Successive passes evaluate pairs of elements, triplets of elements, etc., in the same manner until combinations of one less than the total number of elements have been evaluated. A nontagged element on the first pass results in automatic rejection, and the pattern is given a low rating. Otherwise, the rating is a transformation of the final value of the tally counter. Recognition ratings are, therefore, a function of the probability that the number of tags on an element or combination of elements exceeds the threshold value for incrementing the tally counter and the number of elements and combinations that may be evaluated in that manner, which is in turn determined by the number of elements in the pattern.

The interested reader will have little trouble in determining that there are a number of testable differences between the Reitman and Bower (1973) model and the present model. The matter of primary importance is, however, the common theoretical base from which the models derive. Both models assume that the critical determinant of the "recognition ratings" is not a matter of abstracted prototypes and rules but, instead, the frequency with which certain events occur during acquisition. Both models are apparently successful in predicting the data toward which they are immediately directed. ${ }^{1}$

Some indication of the general applicability of the attribute frequency model may be demonstrated by applying an appropriate version of the model to results of an experiment in the abstraction of linguistic prototypes reported by Bransford and Franks (1971, Experiment I). In this experiment, a prototypical sentence was constructed that consisted of four "ideas." Distortions of this sentence were produced by eliminating one, two, or three ideas from the sentence. The acquisition set was composed of sentences containing three, two, or one idea. The recognition set was composed of similar sentences as well as the prototype. Mean recognition ratings were highest for the prototype and decreased with increasing transformational distance.

An attribute frequency model may be applied to the single stimulus set for which adequate information was reported. The prototype consisted of the sentence "The ants in the kitchen ate the sweet jelly which was on the table." In terms of the attribute frequency model, the attributes may be defined as ants / in kitchen / ate / sweet / jelly / on table. There are two states for each attribute: present and not present; only one of which need be counted. The frequencies with which the individual attributes were present in the sentences that comprised the acquisition set were six, four, four, two, four, and three, respectively. In addition, since syntactic ordering is a powerful cue to relationships among these attributes, the relational frequencies between pairs of
Table 8

Mean Recognition Ratings of Sentences by Transformational Distance and by Derived Frequencies*

\begin{tabular}{|c|c|c|c|c|c|}
\hline $\begin{array}{l}\text { Sentence } \\
\text { Number }\end{array}$ & $\begin{array}{l}\text { Transfor- } \\
\text { mational } \\
\text { Distance }\end{array}$ & Mean & $\begin{array}{r}\text { Sentence } \\
\text { Number }\end{array}$ & $\begin{array}{l}\text { Fre- } \\
\text { quency }\end{array}$ & Mean \\
\hline 1 & 0 & 4.26 & 1 & 21 & 4.26 \\
\hline 2 & 1 & 3.63 & 2 & 20 & 3.63 \\
\hline 3 & 2 & -.73 & 5 & 17 & 3.59 \\
\hline 4 & & 2.93 & 4 & 16 & 2.93 \\
\hline 5 & & 3.59 & 7 & 14 & -.50 \\
\hline 6 & 3 & -2.66 & 3 & 9 & -.73 \\
\hline 7 & & -.50 & 6 & $(-) 6$ & -2.66 \\
\hline
\end{tabular}

*Derived from data reported by Bransford and Franks (1971, Experiment I).

attributes were counted. For example, the frequency with which ants appeared immediately to the left or right of in kitchen was counted, as well as the same relational states for ants and ate, ate and sweet, ate and jelly, sweet and jelly, etc. As with the visual patterns, it was assumed that sentences would be recognized if all relational frequencies were nonzero and that the rating would be commensurate with the sums of the individual attribute frequencies.

Applying this model results in a prediction counter to that of the prototype-plus-transformation model. The sentence numbered 6 by the authors is predicted to be a noncase by the attribute frequency model. This sentence, "The jelly was sweet," places jelly and sweet in a relationship that did not appear in the acquisition set; thus, the relational frequency is zero, predicting a negative rating. The prototype-plus-transformation model assigns the sentence one idea, making it a transformational distance of 3 . Note that, since the sentence is syntactically correct, it could not be judged a noncase on the basis of a syntactical violation caused by the reversal of attributes. Table 8 lists the sentences in the order predicted by the prototype-plustransformation model and the order predicted by the attribute frequency model, as well as the mean ratings reported by Bransford and Franks. The superiority of the attribute frequency model is most striking with respect to its prediction of the negative rating on Sentence 6.

Whether this attribute frequency model can be extended to account for the related results reported by Posner and his colleagues (e.g., Posner, Goldsmith, \& Welton, 1967; Posner \& Keele, 1968) remains to be determined. Their paradigm consists of establishing a visual prototype in the form of a dot pattern either with the shape of a simple, well known symbol, such as a block letter, or in a random pattern. This prototype is then distorted by moving the dots in accordance with a statistical rule. In all cases, recognition is greatest for the prototype and decreases with increased distortion.

Since the dot patterns vary in semicontinuous bidimensional space, there is difficulty in establishing loci at which to count the frequencies of occurrence of 
dots. This does not rule out the possibility of S-defined perceptual sectors of the stimuli. The possible size of these sectors ranges from the complete matrix to sectors the size of a single dot, neither of which extreme yields useful information within the context of the attribute frequency model. Somewhere between the two extremes lies a range of sector sizes in which the frequency of occurrence of the dots should yield predictions as to the mean ratings of the patterns. Posner (1969) noted that, for recognition of patterns composed of a single dot, performance was above chance. He concluded that the density of dots within certain sectors might be a cue to making the correct classification. The density of dots within a certain sector, when extended across stimuli, is synonomous with the frequency of dots occurring in that sector. Thus, it would seem possible to extend the present attribute frequency model to account for results obtained in this paradigm as well.

\section{REFERENCES}

Bourne, L. E., Jr. Human conceptual behavior. Boston: Allyn \& Bacon, 1966.
Bransford, J. D., \& Franks, J. J. Abstraction of linguistic ideas. Cognitive Psychology, 1971, 2, 331-350.

Franks, J. J., \& Bransford, J. D. Abstraction of visual patterns. Journal of Experimental Psychology, 1971, 90, 65-74.

Posner, M. I. Abstraction and the process of recognition. In G. H. Bower and J. T. Spence (Eds.), Psychology of learning and motivation. New York: Academic Press, 1969.

Posner, M. I., Goldsmith, R., \& Welton, K. E. Perceived distance and the classification of distorted patterns. Journal of Experimental Psychology, 1967, 73, 28-38.

Posner, M. I., \& Keele, S. W. On the genesis of abstract ideas. Journal of Experimental Psychology, 1968, 77, 353-363.

Reitman, J. S., \& Bower, G. H. Storage and later recognition of exemplars of concepts. Cognitive Psychology, 1973, 4, 194-206.

\section{NOTE}

1. The author is indebted to James Greeno and Rudolph Schulz for the above observations.

(Received for publication August 17, 1973; revision received October 1, 1973.) 